\title{
A study of the mortality of female asbestos workers
}

\author{
MURIEL L. NEWHOUSE ${ }^{1}, G$. BERRY ${ }^{2}$, J. C. WAGNER ${ }^{2}$, and \\ MARY E. TUROK ${ }^{1}$ \\ London School of Hygiene and Tropical Medicine TUC Centenary Institute of Occupational \\ Health ${ }^{1}$ and the Medical Research Council's Pneumoconiosis Unit, Penarth, Glamorgan²
}

\begin{abstract}
Newhouse, M. L., Berry, G., Wagner, J. C., and Turok, M. E. (1972). Brit. J. industr. Med., 29, 134-141. A study of the mortality of female asbestos workers. A cohort study of over 900 women employed at an asbestos factory making both textiles and insulation materials is described. It extends the information about asbestos-related disease at this factory which was previously available only for male workers. The cohort was defined as all the women who started employment at the factory between 1936 and 1942 and the main analysis was of mortality up to the end of 1968 . This analysis was made in relation to job, length of exposure, and age at first exposure. Compared with national rates there was excess overall mortality among those who worked in jobs with low to moderate exposure partly accounted for by deaths from cancer. In the group with severe exposure, who had worked in the factory for less than two years, there was an excess of cancer of the lung and pleura. However, the most marked increased mortality was in those with severe exposure who had worked for more than two years in the factory; in this group there were excess deaths from cancer of the lung and pleura, from other cancers, and from respiratory diseases. There were no significant trends of excess mortality with age at first exposure. The smoking habits of some of the deceased women were obtained and the indications were that the proportion of smokers in the cohort was higher than the national rate. This could account for some of the excess mortality but the trend of this excess with exposure indicated the role of asbestos. Necropsy reports and/or histological material were obtained for $43 \%$ of those who had died. Three deaths registered as cancer of the pleura were identified as pleural mesothelial tumours; in all there were 11 mesotheliomas, six of pleural and five of peritoneal origin.
\end{abstract}

The mortality of male asbestos workers in mines and factories has been extensively investigated (Doll, 1955; Selikoff, Churg, and Hammond, 1964; McDonald et al., 1971). There is less opportunity to examine the mortality of female workers although there is considerable evidence that women exposed to asbestos are at risk of developing asbestos-related cancers and asbestosis. Keal (1960) reported a high incidence of peritoneal tumours among women patients suffering from asbestosis. Enticknap and Smither (1964) reported three female deaths in their series of 11 patient dying from peritoneal mesothelioma. Wagner, Sleggs, and Marchand (1960) found nine women with mesothelial tumours among 33 subjects in South Africa. Among 83 patients with a confirmed diagnosis of mesothelial tumour from the London Hospital (Newhouse and Thompson, 1965), 42 were females. In Canada, approximately one-third of the mesothelial tumours reported over a 10-year period occurred in women (McDonald, Harper, El Attar, and McDonald, 1970). Mancuso and El-Attar (1967) included 228 women asbestos 
workers in their cohort followed for 25 years; eight of the 31 deaths that occurred during the period were due to asbestosis, six to pulmonary malignancies, and one to a peritoneal mesothelioma.

\section{Definition of the cohort}

Here we describe the mortality experience of women who have been employed in an asbestos factory. Methods of tracing the past employees of this factory have already been described (Newhouse and Williams, 1967). The factory was in production for 56 years and employed more men than women. Reliable information was obtained about a high proportion of the men, but the methods failed to trace more than $30 \%$ of the women employees. The principal difficulty was the change of name on marriage, subsequent to leaving the firm's employment. A further attempt has been made to trace women who started work at the factory during the seven years between 1 January 1936 and 31 December 1942 , a period when the information available to ourselves was expected to link up with the information at the National Health Service Central Register (NHSCR). A pilot study of 100 of the women yielded information on 76 subjects. With this encouraging result the names, dates of birth, and addresses during employment of all 928 women who started work at the factory during this period were sent to the NHSCR. Six hundred and thirty-six $(67 \%)$ members of this cohort were identified as currently alive or, if deceased, a copy of the entry to the Register of Deaths was given. Forty-six (5\%) had either emigrated or joined the armed Forces before 1952 and could not be followed further. A search of the marriage indices at Somerset House identified the married names of a further 63 women whose current status could then be determined by the NHSCR. An attempt was made to obtain information about past workmates from women who had been employed for long periods at the factory. Of the 64 women written to, only 21 replied and four were visited. Information obtained through them and a final search through factory records led to another 17 women being traced. At 31 December 1968,576 of the 928 women were identified as alive and 140 dead. Thus $716(77 \%)$ had been traced. The dates of birth of six of the untraced women were not known and these women were excluded from the cohort at this stage.

\section{Occupational exposure}

One hundred and twenty-six of the 922 women in the cohort had been employed only in jobs with low to moderate exposure to asbestos dust. They were those employed in the office, canteen, laboratory, and print stores. A few with low exposure were in production departments such as brake lining, where chrysotile asbestos was used, and rubber jointings where crocidolite and chrysotile asbestos was used. The remainder had all worked in production departments, over 400 in the textile departments in carding, spinning, doubling or weaving; 100 were in a mattress-making section. These are jobs where there is a statutory requirement under the Asbestos Industry Regulations (1931) for periodic medical examination. Crocidolite was used in the spinning and doubling department and chrysotile and amosite for carding, weaving, and mattress making. Two hundred and twenty-five other women were employed on sectional pipe making, preparing filters for gas masks, and in the yarn store. In these jobs also either crocidolite alone or both crocidolite and amosite were used. Methods of dust suppression were required in all areas, but women in the latter group of jobs were not required to have medical examinations. Workers in both groups of job were considered to be severely exposed to dust. The textile and mattress making departments at this factory closed in the mid 1950s.

\section{Method of analysis}

The results have been assessed by comparing the number of observed deaths with the number of expected deaths. The 'expected' deaths were calculated by the 'man-years' method (Case and Lea, 1955), multiplying years of risk by death rates.

Causes of death, as recorded on the death certificate, were coded according to the Seventh Revision of the International Classification of Diseases (World Health Organization, 1957) and the groups of causes considered were: all cancers (ICD 140-205), cancer of the lung and pleura (ICD 162, 163), cancer of the gastrointestinal tract (ICD 150-157), cancer of the ovary and fallopian tubes (ICD 175), and respiratory diseases excluding lung cancer (ICD 001-008, 240, 241, 465, 490-493, 500502, 518-527).

Death rates for England and Wales were taken from the tables compiled for studies of this kind by Case and his colleagues (General Register Office, 1957; Case and Harley, 1958; Case, Coghill, and Harley, 1968a, b). These tables give age-specific death rates with age in five-year groups up to 85, and calendar year also in five-year groups up to 1961 and 1965 . The period of study extended beyond this and for 1966 to 1968 the rates for 1961 to 1965 were used.

Each woman was considered at risk from the date of first employment at the factory until 31 December 1968 for those traced as alive, the date of death for those known to be dead, or the date of leaving employment at the factory for those untraced.

Excess mortality has been tested by treating the observed number of deaths as a Poisson variable with expectation equal to the man-years expected number of deaths. Although it can be shown that this test may lead to the significance of excess mortality being exaggerated, the size of such a bias is unlikely to be of practical importance. 


\section{Observed and expected mortality}

For the analysis the women were grouped into those with periods of employment lasting up to two years, and those with longer exposure. Each of these groups was further subdivided into two categories, those with low to moderate exposure and those with severe exposure to asbestos dust. The proportion of untraced women varied. It was lowest among women with low to moderate exposure and periods of employment of two years or longer, and highest in the group with severe exposure but shorter periods of employment at the factory (Table 1). A third factor examined was age at start of exposure grouped as 15-24, 25-34, and 35+.

In the total cohort there was no significant excess of deaths with gastrointestinal cancer (observed 11, expected $7 \cdot 3, P=0.12$ ) or cancer of the ovary (observed 4, expected $2 \cdot 1, P=0 \cdot 16$ ) and these two causes have not been detailed in the following mortality tables, although they are discussed where relevant information was obtained.

\section{TABLE 1}

Number OF WOMEN IN COHORT AND NUMBer UNTRACED

\begin{tabular}{|c|c|c|c|c|}
\hline \multirow{3}{*}{$\begin{array}{l}\text { Length of } \\
\text { employment } \\
(y r)\end{array}$} & \multicolumn{4}{|c|}{ Degree of exposure } \\
\hline & \multicolumn{2}{|c|}{ Low to moderate } & \multicolumn{2}{|r|}{ Severe } \\
\hline & No. & No. untraced & No. & No. untraced \\
\hline Less than 2 & 83 & $17(20 \%)$ & 557 & $153(27 \%)$ \\
\hline More than $2 \ldots$ & 43 & $2(5 \%)$ & 239 & $34(14 \%)$ \\
\hline
\end{tabular}

\section{Low to moderate exposure}

Among this group of women there was little difference in the mortality experience of those with short and those with long exposure and, as the group comprised only 126 individuals in all, the results have been combined (Table 2). There was a general excess of deaths which was partly accounted for by deaths from cancer-two from cancer of the lung and pleura and eight from other cancers; this latter group included three gastrointestinal cancers. There was also an excess of deaths from diseases other than cancer or respiratory disease, but this was not significant and did not suggest that any particular cause of death was implicated. The recorded occupations of the two women who died from cancer of the lung were office and canteen worker, jobs which do not suggest an occupational exposure, but nothing is known of possible contact with other workers or exposure in the home.
TABLE 2

MORTALITY OF WOMEN WITH LOW to MODERATE EXPOSURE

\begin{tabular}{|c|c|c|}
\hline \multirow[t]{2}{*}{ Registered cause of death } & \multicolumn{2}{|c|}{$\begin{array}{l}\text { All periods of } \\
\text { employment } \\
\text { (126 women) }\end{array}$} \\
\hline & Obs. & Exp. \\
\hline 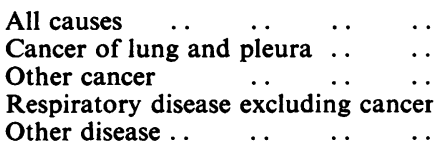 & $\begin{array}{r}29^{1} \\
2^{1} \\
8 \\
2 \\
17\end{array}$ & $\begin{array}{r}18 \cdot 1 \\
0 \cdot 3 \\
4 \cdot 4 \\
2 \cdot 2 \\
11 \cdot 2\end{array}$ \\
\hline
\end{tabular}

${ }^{1 P}<0.05$.

\section{Severe exposure}

This group is larger and is, therefore, divided into those with up to two years' employment and those with longer periods (Table 3). While there was a highly significant excess of deaths from cancer of the lung and pleura in those with the shorter periods of employment, the most marked effect was in those with longer periods of employment. Here there was a significant excess of deaths from cancer of the lung and pleura, other cancers, and respiratory diseases. Among the six dying from cancer of the lung and pleura in the group with short periods of employment were three who were proved to have pleural mesothelioma, one of whom had worked in the factory for only six weeks. The 16 other cancers included five gastrointestinal and one cancer of the ovary. In the group with longer employment, the 17 deaths from other cancers included two registered as dying from peritoneal mesotheliomata, three from gastrointestinal cancer, three from cancer of the

TABLE 3

\section{Mortality of Women With Severe Exposure}

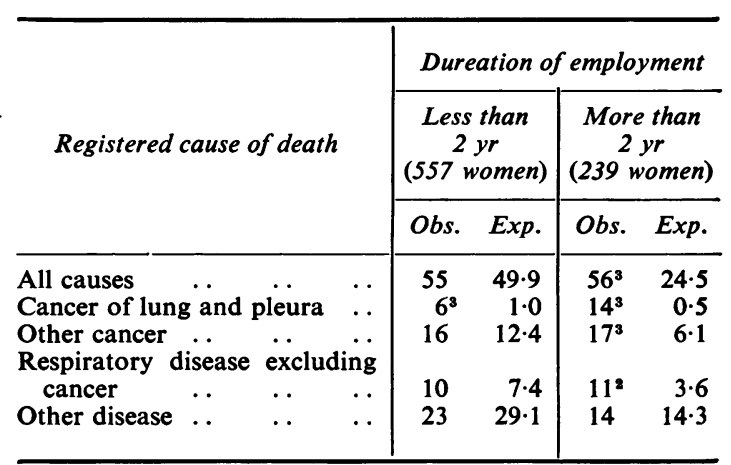

${ }^{2} \mathrm{P}<0.01$.

${ }^{3} \mathbf{P}<0.001$. 
ovary, and three registered as carcinomatosis. In one of the death entries giving carcinomatosis as the primary cause of death, the underlying cause of death was given as 'adenocarcinoma probably bronchus' and in the other two, carcinoma of the ovary was suggested as the primary site of the cancer. In the group of 11 certified as dying from respiratory diseases, the primary cause of death was certified in three as asbestosis.

\section{Excess annual death rate}

In order to obtain a measure of the excess mortality in absolute terms, excess annual death rates were calculated. These rates are obtained by dividing the excess number of deaths (observed minus expected) for any cause by the number of subject-years at risk in the group. For lung cancer, for the group with low to moderate exposure, the mean value of the excess annual death rate was 63 per 100000 years' exposure, for the severely exposed group with short exposure 44, and for the group with severe exposure for two years or longer 241. The differences between the three groups were significant $(P<0.01)$. The third group had a significantly higher excess rate $(P<0.001)$ than the other two.

\section{Effect of age at first exposure}

The mortality was also examined in relation to the age of the women on first employment in the factory (Table 4). Fifty-six per cent started work before the age of 25 and two-thirds of these were under 20; a further $23 \%$ were first employed between the ages of 25 and 34 and $21 \%$ were 35 or older when first engaged. All degrees and lengths of exposure have been combined in Table 4 . In the three age groups the proportions of women in the group with greatest excess mortality, that is those with severe and long exposure, were $27 \%, 21 \%$, and $30 \%$. After adjusting for degree and length of exposure, the mortality pattern is not very different from that given in Table 4 and, hence, conclusions may safely be based on that table. Although the most significant excess mortality occurred in the youngest group, this was due to the larger size of this group and to the lower natural death rates of the younger women. In absolute terms, there are no significant differences in the excess mortality of the three age groups; for lung cancer the excess annual rates were 108, 76, and 122 per 100000 years at risk.

\section{Length of follow-up}

The distribution of observed and expected deaths was also calculated according to the length of followup since first exposure to asbestos in the factory (Table 5). The maximum length of follow-up was 33 years. There was no excess mortality among deaths occurring within 10 years of first exposure. For a follow-up period of between 10 and 20 years, the excess lung cancer mortality was beginning to show, while after 20 years the mortality was much more marked. This is in agreement with the mortality analysis of male asbestos workers (Newhouse, 1969) in which excess mortality was not observed until more than 16 years after first exposure.

\section{Smoking habits}

An attempt was made to ascertain the smoking habits of the women who had died. Little information could be obtained about those who died before 1960 , but from hospital ward notes, general practitioners, and relatives of the deceased it was possible to ascertain the smoking history of 93 out of 103 women who died between 1960 and 1970 . This particular study, therefore, excludes the earlier deaths but includes deaths notified as occurring in 1969 and 1970 . Out of the 93 deceased women, 60 $(65 \%)$ were reported to have been smokers. Taking account of the age distribution of these women and using the smoking prevalences for 1956 for women in the United Kingdom given by Todd (1969), 39 $(42 \%)$ of these women would have been expected to be smokers. However, it is well established that

TABLE 4

Mortality by Age Started Employment at Factory

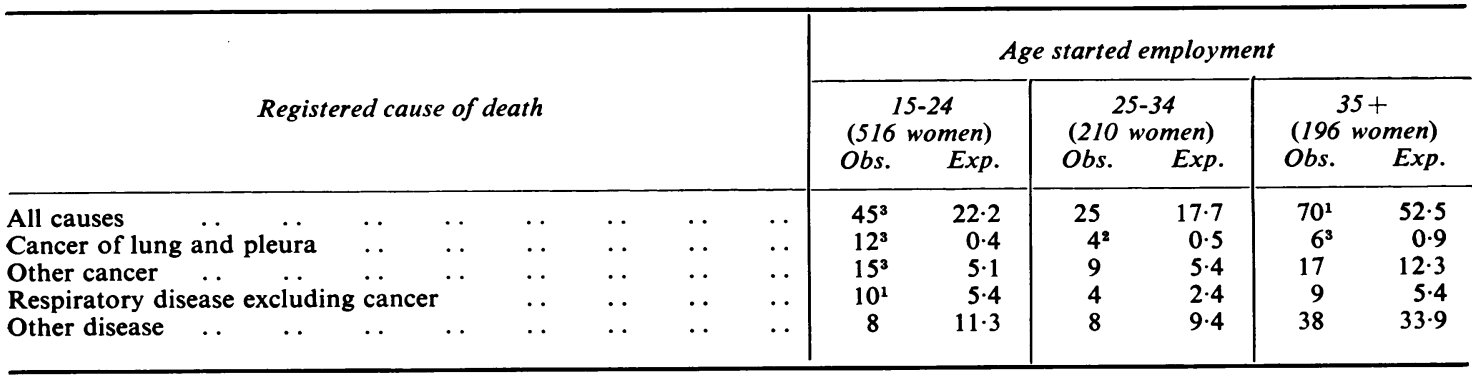

${ }^{\mathbf{I} P<0.05} \quad{ }^{2} \mathrm{P}<0.01 \quad{ }^{3} \mathrm{P}<0.001$ 
TABLE 5

Mortality by LeNGTH of Follow-UP Since First ExPosure

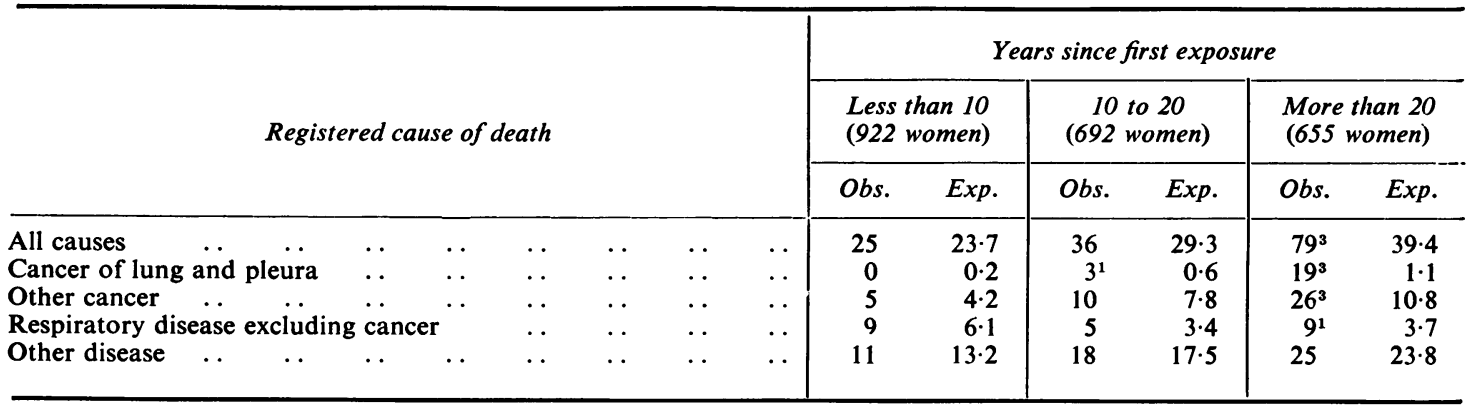

${ }^{1} \mathbf{P}<0.05 \quad{ }^{3} \mathbf{P}<0.001$.

smoking results in a shortening of life (Royal College of Physicians, 1971) so that it would be expected that the proportion of smokers in the above group of deaths would be higher than in the whole cohort. Whether it would have been as high as $65 \%$ if the smoking habits of the cohort were similar to a national rate requires knowledge of the age-specific death-rates for non-smokers and smokers and, as these data are sparse for women, no attempt has been made to examine this point further. It is probable that in this cohort of women there was a higher proportion of smokers than in the whole female population of the United Kingdom.

There were 22 women in the 1960-70 group certified as dying of cancer of the lung and pleura and, of these, six were proved to have a pleural mesothelioma. The smoking habits of these 22 women were four non-smokers, four light smokers ( 1 to 9 cigarettes/day), 10 moderate smokers (10 to 19 cigarettes/day), and four heavy smokers $(20+$ cigarettes/day). Only two of the women who died of pleural mesothelioma were non-smokers.
Validation of the certified cause of death

In this series, as in the series of deaths of male asbestos workers (Newhouse and Wagner, 1969), an attempt was made to obtain information additional to the certified cause of death in order to validate the suggested diagnosis. Hospitals and coroners were approached and asked for copies of postmortem reports, and histological specimens were requested from the pathologists performing the necropsy. In the case of women who had died at home and where the death had been certified as due to cancer of any site, the general practitioner was asked for the name of the hospital where the patient had been treated and a request was made for histological material from biopsy or surgical specimens. There were 140 deaths in the series. In many, particularly where death had occurred before 1950 , records and specimens had not been preserved, but necropsy reports were obtained in 51 (Table 6) and necropsy records together with histological material in 30 of these; biopsy material was obtained in a further nine of the series. The London Hospital and

TABLE 6

Number of Necropsy and Pathological Specimens Received

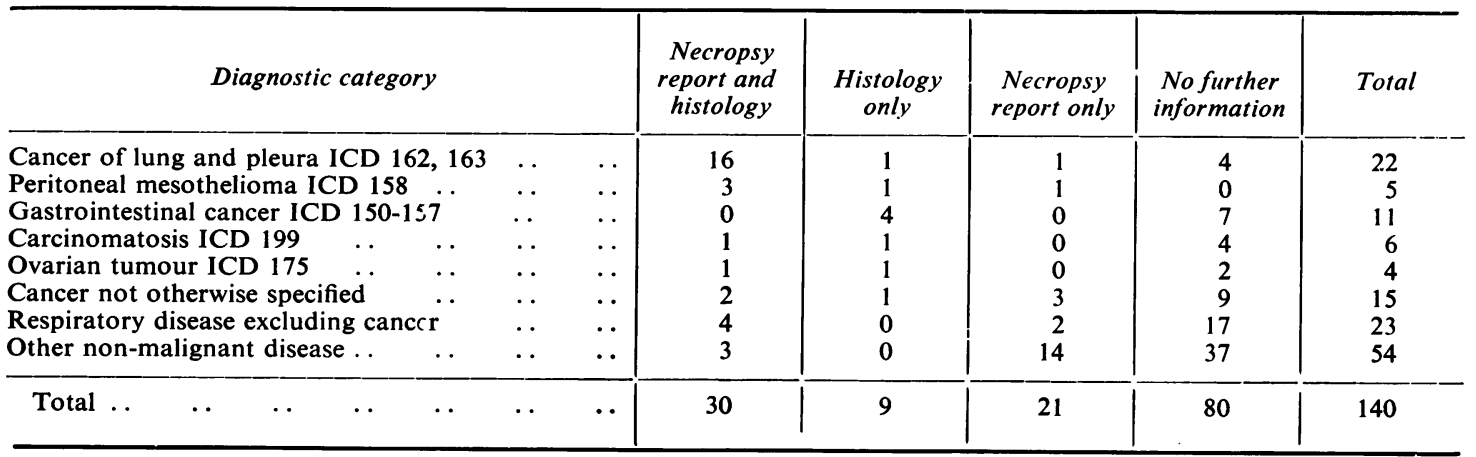


the London Pneumoconiosis Panel together contributed one-third of the necropsy reports and histological material. Eleven necropsy reports came from coroners and the remaining material from 20 other hospitals. All histological material was reviewed by one of us (J.C.W.) and all suspected mesothelial tumours were referred to the members of the mesothelioma panel. The opinion of Dr. F. A. Langley of Manchester University was obtained on tumours of possible ovarian origin.

Additional information was received about all but four of the group of lung cancers (Table 6). Three of the 22 deaths originally certified as cancer of the lung or pleura were re-classified as pleural mesothelioma and this diagnosis was confirmed in a further three cases. There were five deaths certified as peritoneal mesothelioma. This diagnosis was confirmed in each case and no additional tumours were identified.

We were not successful in obtaining any necropsy reports for the 11 women who died of gastrointestinal cancer. Biopsy material was obtained from four; in two, this was adequate to confirm the certified cause of death given as carcinoma of the oesophagus; in the other two, the specimens were too poorly preserved to reveal the tumour type. There were four deaths registered as cancer of the ovary; in the two cases where histological material was available, the registered cause of death was confirmed. Further information was received for only two of the six deaths certified as carcinomatosis. In both cases, examination of the protocols and histology suggested an ovarian origin of the tumour.

Sections of lung tissue were examined in 23 of the series; evidence of asbestosis was found in all but two. The cases for which lung tissue was available for examination included six of those certified as dying of cancer of the lung, and four of pleural mesothelioma, one of peritoneal mesothelioma, and two of asbestosis. In the remaining 10 sections, the certified cause of death was cancer of the breast (2), cerebral tumour (1), uraemia (1), and respiratory conditions (6).

\section{Discussion}

The mortality of the women workers at this factory appears to follow a pattern similar to that of the male workers (Newhouse, 1969). In both groups there is a highly significant excess of cancers of the lung and pleura. Among workers followed for more than 20 years, about $1 \%$ of the men and $1.5 \%$ of the women died of mesothelial tumours. However, in this series, only $77 \%$ of the members of the cohort were traced. If the 206 untraced women had experienced a similar mortality to the traced, then the expected number of deaths among them would be about 29. If the true number of deaths was very much different from this, then the incompleteness of follow-up would have introduced a bias. As an extreme example, suppose none of the untraced women had died, then the expected numbers of deaths would be approximately: total deaths 113 (observed 140), all cancers 30 (observed 63), lung cancer 2.2 (observed 22). Hence, even in this highly unlikely event, there would still have been excess mortality. The method of tracing was such that the fact that a woman had died did not materially increase the likelihood of her being traced.

The use of rates for 1961-65 for calculating expected deaths for 1966-68 may be criticized, but examination of age-specific death rates for 1962, 1963, and 1967 (General Register Office, 1964-70) showed the error in the calculated number of expected deaths to be about $1 \%$ or less for total deaths and all separate causes except for lung cancer. Here there was an increase of $25 \%$ in the rates between 1963 and 1967 which modified the expected number of deaths by $5 \%$; however, this results in the negligible change from 1.8 to 1.9 in the expected figure. Secondly, the uses of rates from a smaller and geographically simila: area such as Greater London might have been more appropriate than the national rates. The SMR for lung cancer for Greater London was $137 \%$ in the quinquennia 1963-67 (General Register Office, 1964-70). If this figure had been applied to the expected number $(1 \cdot 8)$, we would have expected 2.4 deaths. This would still have been a highly significant excess.

Eleven mesothelial tumours were identified, six pleural and five peritoneal. Six of these tumours occurred in women who had worked for less than two years in the factory. The proportion of peritoneal tumours is high compared with several series of necropsy cases. For example, McEwen, Finlayson, Mair, and Gibson (1970), in a Scottish study of a predominantly male population, found only three cases arising from the peritoneum out of 80 mesotheliomas; Ashcroft and Heppleston (1970) found three out of 23 in a Tyneside study, and Thomson (1970) found three out of 17 in Cape Town. Howeve; , higher proportions of peritoneal tumours have been found in cohort studies of asbestos workers. In the study of males from the same factory as the female workers, 13 peritoneal mesotheliomas out of a total of 19 were reported (Newhouse and Wagner, 1969) and more recent figures for this study are 30 out of 49. Selikoff, Hammond, and Churg (1970) found 16 peritoneal mesotheliomas out of a total of 22 mesotheliomas occurring in their cohort of male asbestos insulation workers in the New York area, and these authors suggested that the differences between the two types of study could be the result of the greater exposure in the two cohort studies, pleural mesotheliomas being associated with lesser exposure and peritoneal with greater. 
There were four deaths in all registered as cancer of the ovary; three of these occurred among the women with severe and long exposure and, compared with the expected number of 0.6 in this particular group, this was a significant finding $(\mathbf{P}=$ 0.0025). The histological review suggested that at least two of the other deaths in the group which were registered as carcinomatosis were due to this cause. These figures are too small for definite conclusions but, in view of the experimental work (Graham and Graham, 1967) which suggested that ovarian tumours can be produced in laboratory animals after the intraperitoneal injection of tremolite asbestos, collection of further data is of importance.

There was a highly significant excess of deaths from cancer of the lung occurring chiefly in those with severe exposure to asbestos dust. As in the study of male asbestos workers (Newhouse, 1969), it was found in both the group with short and the group with long exposure, but the excess lung cancer risk which was found in the low-moderate exposure group was not observed in the male study. This difference could be due to the suggested higher proportion of smokers in tne cohort than in the general female population. Our evidence on smoking is incomplete and obtained at second hand, but Todd (1966) found that recalls of smoking habits by relatives were a reasonably accurate substitute for recalls by the smokers themselves. The much higher excess lung cancer risk in those women with severe exposure for a shorter period and the occurrence of pleural mesotheliomas confirms that the exposure to asbestos was responsible for an appreciable proportion of the excess lung cancers. Comparisons within the severe exposure group also show that asbestos exposure resulted in excess deaths from other cancers and deaths with respiratory diseases.

The excess of lung cancer deaths is much more striking in the women than in the men (Newhouse, 1969). In the severely exposed group the ratio of observed to expected lung cancer is 13 for the women but only $2 \cdot 5$ for the men. Jacob and Anspach (1965) found a similar result with ratios of 16 and $2 \cdot 1$ for women and men respectively. However, if one considers the absolute excesses the rates are similar for men and women and it is, therefore, suggested that exposure to asbestos produces a similar effect in men and women as far as lung cancer is concerned, but that the effect is more easily detected in a cohort of women because of the lower lung cancer rates in the general population.

The presentation in Table 4 of the effect of age at first exposure may be criticized in that no allowance was made for the length of follow-up which we have shown to be important. We would expect that the average length of follow-up would decrease with age at first exposure because of the higher mortality of older women and that Table 4 would therefore underestimate an age effect. As another way of looking at this aspect, length of follow-up has been allowed for by apportioning the excess number of lung cancers for each length of follow-up between the three age groups according to the number of subject years. For the 15-24 year group this gave an expected excess of 12.2, for the 25-34 year group of $4 \cdot 6$, and for the $35+$ group of $3 \cdot 4$. The observed excesses were $11 \cdot 6,3 \cdot 5$, and $5 \cdot 1$, which are $95 \%$, $76 \%$, and $150 \%$ of the expected figures. Therefore, in the oldest group there is some support of an age effect on mortality from lung cancer. However, the trend, in contrast to the findings of Knox, Holmes, Doll, and Hill (1968), is not at all significant. Evidence on the effect of age at first exposure has recently been reviewed by Doll (1970) and found to be conflicting.

Selikoff, Hammond, and Churg (1968) found that male asbestos workers with a history of regular cigarette smoking had about 90 times the risk of dying of bronchogenic carcinoma as men who neither smoked cigarettes nor were occupationally exposed to asbestos dust. More information is needed on the combined effect of smoking and asbestos exposure on mortality and our study of both male and female workers is being extended in co-operation with the Department of Health and Social Security by carrying out a survey of the smoking habits of the surviving individuals in both cohorts. When this is complete, it may be possible to estimate how much of the excess risk of dying from lung cancer is due to smoking and how much is due to asbestos exposure. The cohort study is also continuing, notification of deaths being received within a short period of their occurrence. More information, both about the effect of smoking in asbestos-exposed workers and about the occurrence of other possibly asbestos-related tumours, should be available within the next 10 years.

We are grateful to Dr. W. J. Smither and the Cape Asbestos Company for their invaluable assistance and co-operation; to the Registrar General and the National Health Central Registrar for supplying copies of the death entries and for tracing the subjects in the study; to Dr. F. A. Langley for examining some of the pathological material; and to Dr. J. C. Gilson and Professor R. S. F. Schilling for their advice and encouragement.

The study was undertaken with the assistance of a grant to M.L.N. from the Medical Research Council.

\section{References}

Asbestos Industry Regulations (1931). Asbestos Industry Regulations: Statutory Rules and Orders, 1931, No. 1140. H.M.S.O., London.

Ashcroft, T., and Heppleston, A. G. (1970). Mesothelioma and asbestos on Tyneside-a pathological and social study. In Pneumoconiosis. Proceedings of the International Conference, Johannesburg 1969, edited by H. A. Shapiro, pp. 177-179. Oxford University Press, Cape Town. 
Case, R. A. M., Coghill, C., and Harley, J. L. (1968a). Supplement to cancer death rates by site, age and sex, England and Wales. Death rates for $1956-1960$ and 1961-1965. Chester Beatty Research Institute, London.

- , - and - (1968b). Supplement to death rates by age and sex for tuberculosis and selected respiratory disease, England and Wales, 1911-1955. Death rates for 1956-1960 and 1961-1965. Chester Beatty Research Institute, London.

- and Harley, J. L. (1958). Death rates by age and sex for tuberculosis and selected respiratory diseases, England and Wales, 1911-1955. Chester Beatty Research Institute, London.

_ and Lea, A. J. (1955). Mustard gas poisoning, chronic bronchitis, and lung cancer. Brit. J. prev. soc. Med., 9, 62-72.

Doll, R. (1955). Mortality from lung cancer in asbestos workers. Brit. J. industr. Med., 12, 81-86.

(1970). Cancer and ageing: the epidemiological evidence. Dorn Memorial Lecture, 10th International Conference of the International Union against Cancer, Houston, U.S.A., May 1970.

Enticknap, J. B., and Smither, W. J. (1964). Peritoneal tumours in asbestosis. Brit. J. industr. Med., 21, 20-31.

General Register Office (1957). Studies on medical and population subjects, no. 13. Cancer statistics for England and Wales, 1901-1955 by A. McKenzie, with additional material by R. A. M. Case and J. T. Pearson. H.M.S.O., London.

- (1964-1970). The Registrar General's statistical review of England and Wales for the years 1962-1968. Part 1, tables, medical. H.M.S.O., London.

Graham, J., and Graham, R. (1967). Ovarian cancer and asbestos. Environ. Res., 1, 115-128.

Jacob, G., and Anspach, M. (1965). Pulmonary neoplasia among Dresden asbestos workers. Ann. N.Y. Acad. Sci., 132, 536-548.

Keal, E. E. (1960). Asbestosis and abdominal neoplasias. Lancet, 2, 1211-1216.

Knox, J. F., Holmes, S., Doll, R., and Hill, I. D. (1968) Mortality from lung cancer and other causes among workers in an asbestos textile factory. Brit. $J$. industr. med., 25, 293-303.

McDonald, A. D., Harper, A., El Attar, O. A., and McDonald, J. C. (1970). Epidemiology of primary malignant mesothelial tumors in Canada. Cancer (Philad.), 26, 914-919.

McDonald, J. C., McDonald, A. D., Gibbs, G. W., Siemiatycki, J., and Rossiter, C. E. (1971). Mortality in the chrysotile asbestos mines and mills of Quebec. Arch. environm. Hlth, 22, 677-686.
McEwen, J., Finlayson, A., Mair, A., and Gibson, A. A. M. (1970). Mesothelioma in Scotland. Brit. med. J., 4, 575-578.

Mancuso, T. F., and El-Attar, A. A. (1967). Mortality pattern in a cohort of asbestos workers. A study based on employment experience J. occup. Med., 9, 147-162.

Newhouse, M. L. (1969). A study of the mortality of workers in an asbestos factory. Brit. J. industr. Med., 26, 294-301.

- and Thompson, H. (1965). Mesothelioma of pleura and peritoneum following exposure to asbestos in the London area. Brit. J. industr. Med., 22, 261-269.

- , and Wagner, J. C. (1969). Validation of death certificates in asbestos workers. Brit. J. industr. Med., 26, 302-307.

—, and Williams, J. M. (1967). Techniques for tracing past employees; an example from an asbestos factory. Brit. J. prev. soc. Med., 21, 35-39.

Royal College of Physicians (1971). Smoking and Health Now, pp. 24-34. Pitman Medical and Scientific, London.

Selikoff, I. J., Churg, J., and Hammond, E. C. (1964). Asbestos exposure and neoplasia. J. Amer. med. Ass., $188,22-26$.

- Hammond, E. G., and Churg, J. (1968). Asbestos exposure, smoking, and neoplasia. J. Amer. med. Ass., 204, 106-112.

, - and - (1970). Mortality experience of asbestos insulation workers 1943-1968. In Pneumoconiosis. Proceedings of the International Conference, Johannesburg, 1969, edited by H. A. Shapiro, pp. 180-186. Oxford University Press, Cape Town.

Thomson, J. G. (1970). The pathological diagnosis of malignant mesothelioma of pleura and peritoneum. In Pneumoconiosis. Proceedings of the International Conference, Johannesburg 1969, edited by H. A. Shapiro, pp. 150-154. Oxford University Press, Cape Town.

Todd, G. F. (1966). The Reliability of statements about smoking habits. Supplementary Report. Research paper no. 2a, Tobacco Research Council, London.

- (1969). Statistics of Smoking in the United Kingdom. Research paper no. 1, 5th ed., p. 26, Tobacco Research Council, London.

Wagner, J. C., Sleggs, C. A., and Marchand, P. (1960). Diffuse pleural mesothelioma and asbestos exposure in North Western Cape Province Brit. J. industr. Med., 17, 260-271.

World Health Organization (1957). Manual of the International Statistical Classification of Diseases, Injuries, and Causes of Death. W.H.O., Geneva.

Received for publication on July 19, 1971. 